\title{
Time structure analysis of extensive air showers using the Telescope Array Data
}

\author{
R. Mayta*, Y. Tsunesada, S. Ogio for the Telescope Array Collaboration ${ }^{\dagger}$ \\ Graduate School of Science, Osaka City University \\ E-mail: d17sa301@vh.osaka-cu.ac.jp
}

\begin{abstract}
In this paper, we report results of the study of temporal shower front structure based on the data taken by the Telescope Array Surface Detector from 2008 to 2019. By analyzing the waveforms measured by FADCs, we defined two quantities characterizing the shower structure, the curvature of the shower front and the thickness of the shower disk. These analyses were performed by selecting the data sample of high-quality events at zenith angles up to 60 degrees. Firstly we used an empirical form of the shower front curvature in term of residual time, and found a clear zenith angle dependence but no significant energy dependence based on the 3 energy intervals in $\log (\mathrm{E} / \mathrm{eV})$ bins from 18.90 to 20.00 . Secondly, the thickness of the disk is analyzed by the rising time of the FADC waveform. We found that the thickness of the shower disk has a dependency on angles and energy.
\end{abstract}

36th International Cosmic Ray Conference -ICRC2019-

July 24th - August 1st, 2019

Madison, WI, U.S.A.

\footnotetext{
${ }^{*}$ Speaker.

${ }^{\dagger}$ for collaboration list see PoS(ICRC2019)1177
} 


\section{Introduction}

Cosmic rays are crucial clue to understand high-energy phenomena in the universe. Cosmic rays interact with air, then a phenomenon so-called "air shower" produces many daughter particles. The Telescope Array has been designed to study cosmic rays with energy greater than $1 \mathrm{EeV}$ by using the fluorescence detectors (FD) and the surface detectors (SD) to detect air showers. The Telescope Array experiment is operating since its complete layout in 2008 allowing a complete and detailed three-dimensional reconstruction of shower front with high space-time resolution. The air shower structure depends on energy, arrival direction, primary mass and on the interaction mechanism with air nuclei. Several techniques would be required to well understand in details the air shower properties. In this context, shower parameters are studied by using the TA-SD data.

The TA-SD array consists of 507 detectors arranged in a square grid on the ground with $1.2 \mathrm{~km}$ spacing. Each detector contains 2 layers of plastic scintillator and each layer has an area of $3 \mathrm{~m}^{2}$ and a thickness of $1.2 \mathrm{~cm}$. The two output signals from PMTs are digitized by $12 \mathrm{bit}$ Flash Analog to Digital Converters (FADC) with a $50 \mathrm{MHz}$ sampling rate. The time window range of waveform is minimum $2.56 \mu \mathrm{s}$. The judgement of signal recording is based on 2 types of low level triggers, first is when the signals are greater than 0.3 minimum ionizing particles (MIP) as level-0 trigger and second signals greater than 3.0 MIPs are stored as level-1 trigger event. If three or more adjacent SDs announce level-1 triggers within an $8 \mu$ s window, this establishes a level- 2 event trigger. All level-0 trigger waveforms within $\pm 32 \mu$ s of the event are stored [2][8]. TA has taken data for 11 years from 2008-05-11 to 2019-05-10, using that 11 years of the SD data, we analyzed the shower structure to understand two properties of air showers. At first the curvature of the shower front as the residual time with respect to the planar fit. And the second observable is the thickness of the shower disk which is defined as the rise time from $10 \%$ to $50 \%$ of the integral signal waveform. We present the results of the shower properties with energy and arrival direction dependencies.

\section{Data set and selection rules}

The surface detector event reconstruction follows two main steps: first the event geometry is determined by using the arrival time of the shower front particles measured by counters that triggered in the course of the event and by SDs that have sufficient signal to noise ratio. The direction and the shower core position of the primary particle are reconstructed. Second, from the pulse height signals processed and calibrated in units of VEM in these counters, along with the event geometry information, are used for determining the shower lateral distribution profile. More reliable reconstruction of the shower core position and the scaling factor $\mathrm{N}$ are reconstructed from the Eq. 4.5 [1]. In the following we describe the selection rules applied in this shower structure analysis:

- Zenith angle cut: $0^{\circ}<\theta<60^{\circ}$

- Border cut: $1.2 \mathrm{~km}$ inside of array

- Geometry and Lateral Distribution Function (LDF) fit quality cut, $\chi^{2} /$ d.o.f. $<4$

- Numbers of SD triggered 6 or more 
- Shower front fitting quality (Sec. 4.1) $\chi^{2}<20$.

Quality cut in the thickness of the shower was also studied based on the total recorded signal. In all analysis of shower structure we use $\zeta$ angles definition, it is defined as the angle around the shower axis. The direction where the shower front hits the ground earliest is defined $\zeta=0^{\circ}$ and the direction where the shower front hits the ground latest is defined $\zeta=180^{\circ} /-180^{\circ}$. In this analysis, the positive $\zeta$ is defined in the anticlockwise direction looking from above.

\section{Time structure}

The TA-SD array can measure the particle arrival times and the number of particles in term of the pulse height per unit area $\left(\mathrm{VEM} / \mathrm{m}^{2}\right)$ as density, $\rho$, at the ground. Based on the information of each SD counter and more precisely time profile, we describe the analyses of 1) the curvature of the shower front by using the definition of residual time with respect to the shower plane and 2) the thickness of the shower disk by analyzing the observable rise time based on the accumulated waveforms. Figure 1 illustrates the scheme for air shower measurement with the surface detectors.

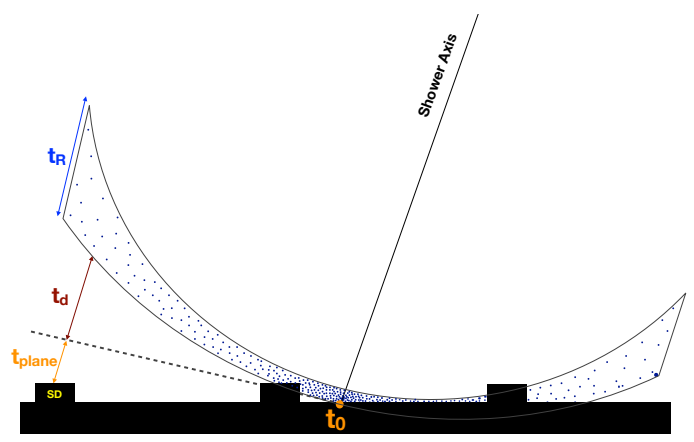

Figure 1: Scheme of shower front with the representation of the residual time $\left(t_{d}\right)$ and the thickness of the shower disk as $\left(t_{R}\right)$.
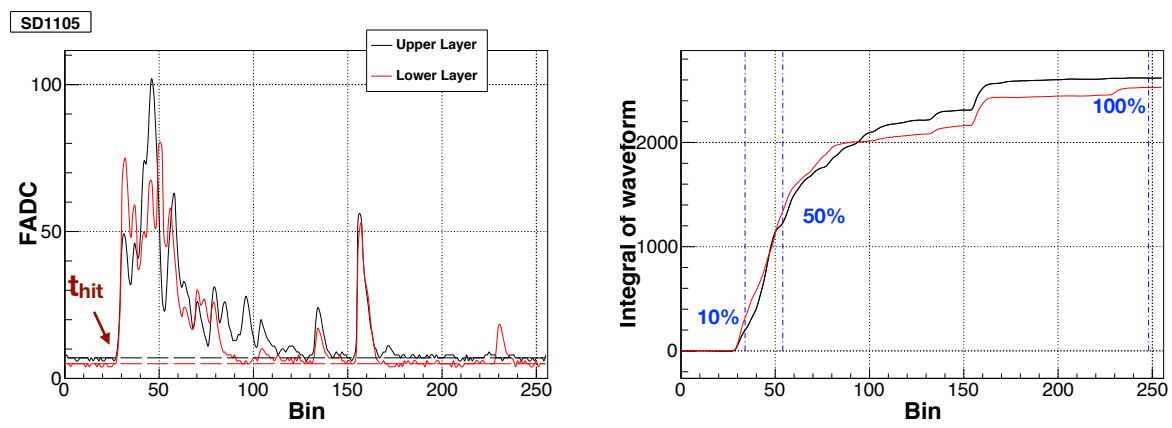

Figure 2: (Left) Typical FADC waveforms of a TA-SD counter as a function of time-bin. The black and red lines represent the upper and lower layers response signals of PMTs of one SD. (Right) The accumulated waveforms of the left figure.

The residual time with respect to the shower front plane is represented by $t_{d}$ and the disk thickness 
is represented by the rise time $t_{R}$.

The timing of the shower axis hit on the ground $t_{0}$ is a part of the event, and the other variables $t_{R}$, $t_{d}, t_{\text {plane }}$ in Fig. 1 , are defined for the $i^{t h} \mathrm{SD}$ detector. The $\mathrm{SD} t_{\text {plane }}$ is defined as $l / c$, where $l$ is the distance from the shower plane to the SD along the shower axis direction, $c$ is the speed of light. To analyze these two observables $t_{d}$ and $t_{R}$, we extract the information from the waveform response of each SD counter. Figure 2 in the left panel describes typical FADC counts versus number of time-bin relation, where 1 bin is equivalent to $20 \mathrm{~ns}$, and one typical waveform covers $2.56 \mu \mathrm{s}$. Here the hit time $t_{h i t}$ is defined as the time at which the signal level exceeds the threshold of 0.3 MIP from the baseline. Each SD counter has two PMTs corresponding to the upper and lower layer scintillators. The black and red lines in Fig.2 (left) represent the upper and lower layers, respectively. In the study of the shower front, we use only the hit time $t_{\text {hit }}$ of each waveform, and in case of the thickness of the shower disk we analyze all waveforms information of the shower footprint by using upper layer.

\section{Shower front curvature analysis}

\subsection{Curvature shower analysis procedure}

The shower analysis procedure is based on an iterative process to find the time at core location $t_{0}$. Then $t_{0}$ was obtained by minimizing the function,

$$
\chi^{2}=\frac{1}{n-3} \sum_{i}^{n} \frac{\left(t_{h i t, i}-t_{0}^{\text {fit }}-t_{\text {plane }, i}-t_{d, i}\right)^{2}}{\sigma_{S D}^{2}+t_{\sigma, i}^{2}} .
$$

In equation 4.1, the starting value of $t_{0}^{f i t}$ is when the density, $\rho_{i}$ is big, the scanning of $t_{0}$ is within $\pm 1 \mu \mathrm{s}$ with a step of $0.1 \mu \mathrm{s}$.

When we fit by using the starting $t_{d, i}$ and $t_{\sigma, i}$ functions, which are the modified Linsley time residual and its uncertainty functions [5][6], with the shower front curvature parametrized as a function of zenith angle:

$$
\begin{aligned}
& t_{d, i}=\left(8 \times 10^{-4} \mu \mathrm{s}\right) a(\theta)\left(1+\frac{R_{i}}{30 m}\right)^{1.5} \rho_{i}^{-0.5} \\
& t_{\sigma, i}=\left(7 \times 10^{-4} \mu \mathrm{s}\right) a(\theta)\left(1+\frac{R_{i}}{30 \mathrm{~m}}\right)^{1.5} \rho_{i}^{-0.3} \\
& a(\theta)=\left\{\begin{array}{lr}
3.3836-0.018148 \theta & : \theta<25 \\
c_{3} \theta^{3}+c_{2} \theta^{2}+c_{1} \theta+c_{0} & : 25^{\circ} \leq \theta<35^{\circ} \\
\exp \left(-3.2 \times 10^{-2} \theta+2.0\right) & : \theta>35^{\circ}
\end{array}\right.
\end{aligned}
$$

where $c_{0}, c_{1}, c_{2}$ and $c_{3}$ are constants,

$$
\begin{aligned}
& c_{0}=-7.761668 \times 10^{-2}, c_{1}=2.99113 \times 10^{-1} \\
& c_{2}=-8.79358 \times 10^{-3}, c_{3}=6.51127 \times 10^{-5} .
\end{aligned}
$$

And $\sigma_{S D}$ is the uncertainty due to electronics which, is $20 \mathrm{~ns}$.

After $t_{0}$ was determined, the residual time $t_{d}$ of the particles was observed by each detector SD from the plane shower front of the shower particles. The relation $t_{d}$ and $\mathrm{R}$ distance from shower axis was analyzed for different $\sec \theta$ interval bands. An example of observed shower front in interval $\sec \theta$ : $1.0-1.2$ is shown in Fig.3. 


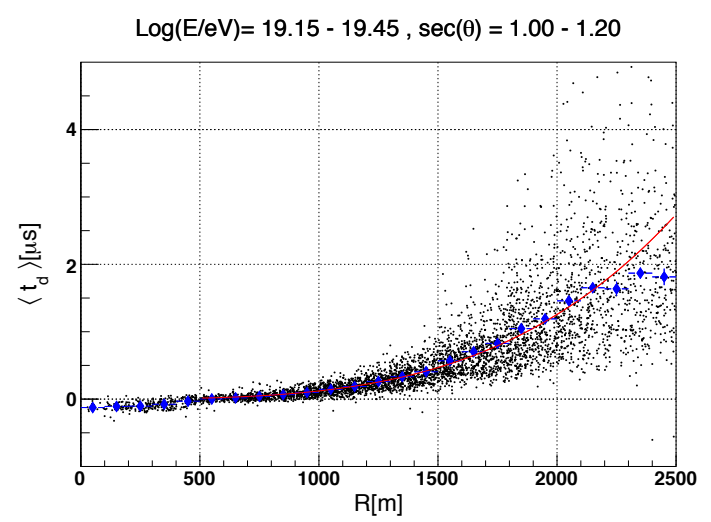

Figure 3: Example of shower front vs core distance distribution for each SD counters in an interval of energy of $10^{19.15}-10^{19.45} \mathrm{eV}$ and zenith angle interval from 0 to 33.6 degrees.

In this analysis, we used the residual time $t_{d}$ introduced by the AGASA experiment [4] and it is expressed in the simple form,

$$
t_{d}=2.6 \times\left(1+\frac{R}{30 \mathrm{~m}}\right)^{A} \times \rho^{B}\left[m^{-2}\right][n s]
$$

here $\mathrm{A}$ and $\mathrm{B}$ are 1.5 and -0.5 respectively determined by the AGASA experiment. And the particle density $\rho$ is fitted by the lateral distribution function:

$$
\rho=N\left(\frac{R}{91.6 \mathrm{~m}}\right)^{-1.2}\left(1+\frac{R}{91.6 \mathrm{~m}}\right)^{-(\eta(\theta)-1.2)}\left(1+\left[\frac{R}{1000 \mathrm{~m}}\right]^{2}\right)^{-0.6}
$$

where $\eta(\theta)=3.97-1.79[\sec (\theta)-1]$.

In our analysis of shower front, we studied the parameters A and B of Eq. 4.4 using the TA-SD data. Figure 3 shows a scatter distribution of the residual time as a function of R, denoted by the black dots which correspond each counter residual time and the blue points represent the average of $\left\langle t_{d}\right\rangle$ with a $100 \mathrm{~m}$ binning. The red line is the function fitting of AGASA from 500 to $2500 \mathrm{~m}$. This example represents the curvature of the shower front for average energy of $\log (E / e V)=19.3$ for showers with zenith angles from 0 to 33.6 degrees.

\subsection{Parameters of the shower front curvature}

Parameters of the shower front curvature are analyzed in the three energy intervals, the lower energy average is $10^{19.0} \mathrm{eV}$, the second is $10^{19.3} \mathrm{eV}$ and the highest energy is $10^{19.8} \mathrm{eV}$. The parameters $\mathrm{A}$ and $\mathrm{B}$ in the AGASA residual time function were obtained using the TA-SD data. Fig.4 shows the parameters $\mathrm{A}$ and $\mathrm{B}$ as a function of $\sec \theta$. The black, blue and red points represent the results using the data sets with the average energies of $10^{19.0} \mathrm{eV}, 10^{19.3} \mathrm{eV}$ and $10^{19.8}$ respectively. In case of the parameter $A$ there is a clear dependence on $\sec \theta$ and contrarily the parameter B has no dependence on $\sec \theta$.

$\operatorname{Sec} \theta$ dependence of $\mathrm{A}$ is fitted by a linear function and summarized below in the three energy intervals together with the constant values of B. Only statistical errors are presented. 

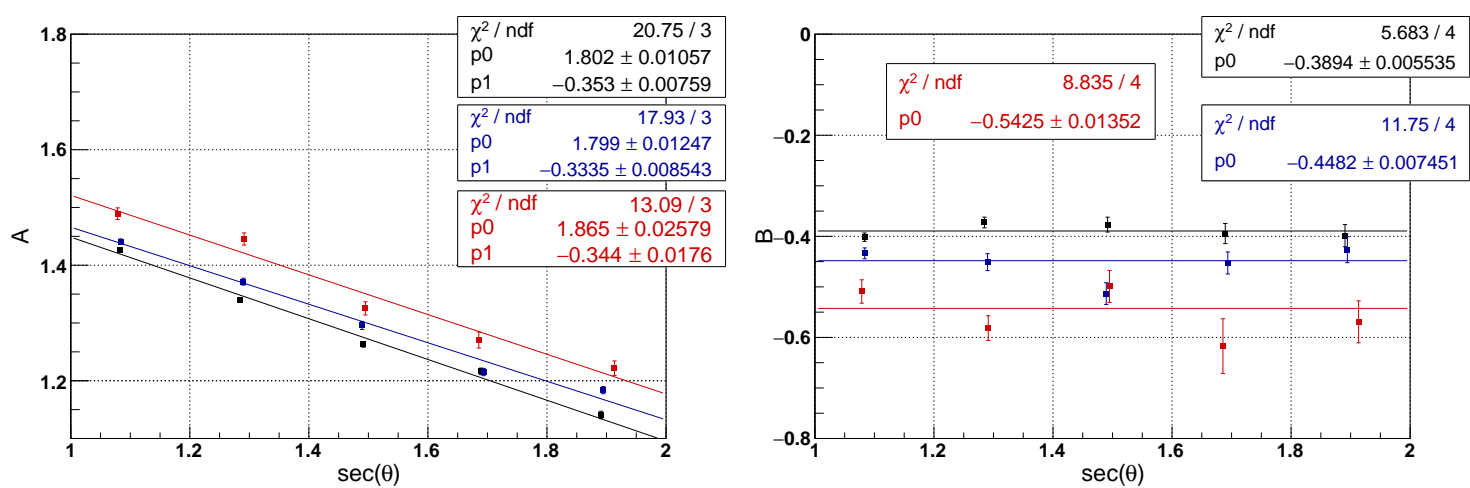

Figure 4: Parameters $A$ and $B$ as function of $\sec \theta$, black, blue and red square dots represent for average energies in $\log (\mathrm{E} / \mathrm{eV}) 19.0,19.3$ and 19.8 respectively. Parameter A represents the power law of curvature shower and $\mathrm{B}$ is particle density parameter.

- $\langle\log (\mathrm{E} / \mathrm{eV})\rangle=19.0$

$A=(1.802 \pm 0.010)-(0.353 \pm 0.008) \times \sec \theta$ and $B=-0.389 \pm 0.006$

- $\langle\log (\mathrm{E} / \mathrm{eV})\rangle=19.3$

$A=(1.799 \pm 0.012)-(0.334 \pm 0.01) \times \sec \theta$ and $B=-0.448 \pm 0.007$

- $\langle\log (\mathrm{E} / \mathrm{eV})\rangle=19.8$

$A=(1.865 \pm 0.026)-(0.344 \pm 0.018) \times \sec \theta$ and $B=-0.542 \pm 0.013$

\section{Shower disk thickness analysis}

\subsection{Definition of the rise time}

The thickness of the shower disk is defined as the width of a certain time of accumulated waveform. In this study we use the rise time defined as the time interval in which the accumulated waveform reaches from $10 \%$ to $50 \%$ of the total SD signal as represented in the right panel of Fig.2. An example of the rise time as a function of the distance from the shower core is shown in Fig.5. As it can be seen, the rise time increases as $\mathrm{R}$ increases. The black dots show the rise time and the blue diamond dots are the average of rise time with a $100 \mathrm{~m}$ binning. This example represents the rise time distribution for events with average energy of $\log (\mathrm{E} / \mathrm{eV})=19.3$ for vertical showers with zenith angles from 0 to 33.6 degrees. The average of rise time (blue diamond points) was fitted with a linear function. $\left\langle t_{R}\right\rangle=b+a \times R$ as a first approximation in an interval of 500 to $1200 \mathrm{~m}$ of distance from the core $\mathrm{R}$.

\subsection{Parameters of the shower disk thickness}

The relations between $\mathrm{R}$ and $t_{R}$ are studied in 5 intervals of $\sec \theta$ and 3 intervals in the primary energy. The parameter $a \mathrm{~ns} / \mathrm{m}$ as a function of $\sec \theta$ is shown in Fig.6 (left). The results from the events in the energy average $\log (\mathrm{E} / \mathrm{eV})=19.0,19.3,19.8$ are shown by the black, blue and red points, respectively. A clear $\sec \theta$ dependence of the $a$ parameter is found. These results describe the properties of the showers age. To study this shower age effect in more detail, as an example we 


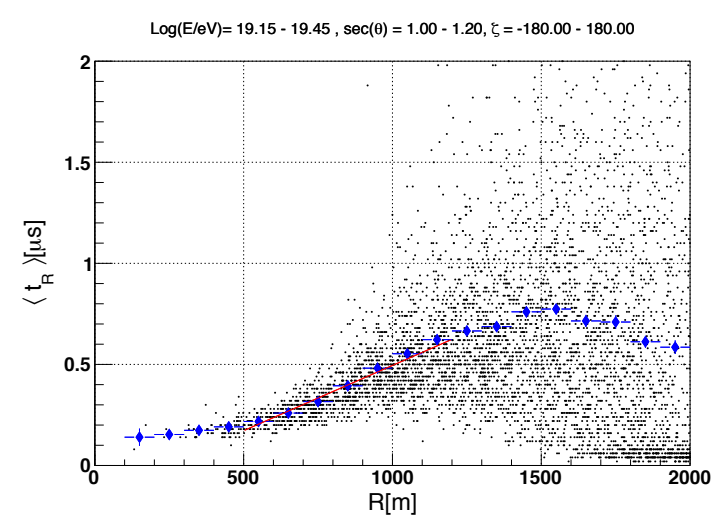

Figure 5: Rise time vs core distance for each SD counter in an interval of energy of $10^{19.15}-10^{19.45} \mathrm{eV}$ and zenith angle interval from 0 to 33.6 degrees. Each black dot is rise time of SD counter and blue diamond dots represent the average of rise time $\left\langle t_{R}\right\rangle$ in bin-by-bin of $100 \mathrm{~m}$.
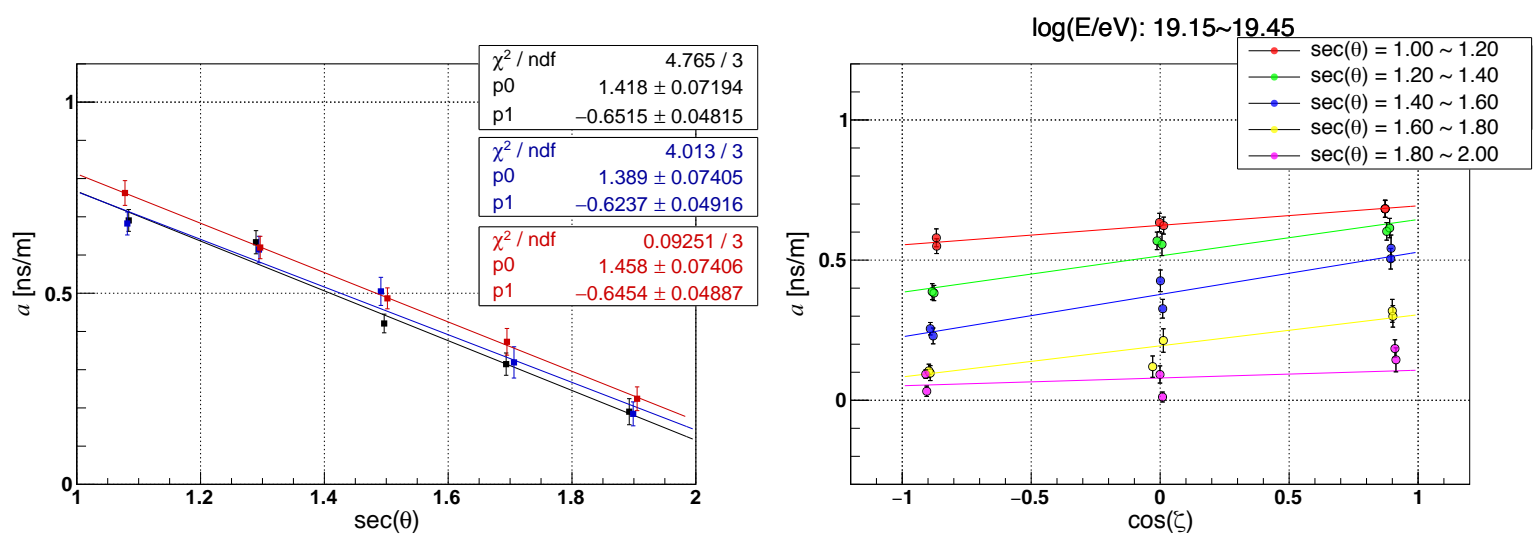

Figure 6: Left panel represent the parameter of thickness in terms of slope $a[\mathrm{~ns} / \mathrm{m}] \mathrm{vs} \sec \theta$, the black, blue and red square dots represent for energies $\log (\mathrm{E} / \mathrm{eV}): 19.0,19.3$ and 19.8 respectively. Right panel shown the dependence of slope $a[\mathrm{~ns} / \mathrm{m}]$ on $\cos \zeta$ in the shower plane for primary energy $\log (\mathrm{E} / \mathrm{eV})=19.30$ in average at different $\sec \theta$ intervals.

show in Fig.6 (right) the values of the thickness parameter $a$ as a function of $\cos \zeta$ for 5 intervals of $\sec \theta$ in step of 0.2 for an interval of energy centered at $\log (E / e V)=19.30$. For each $\sec \theta$ interval band the data are fitted with a function $a=a_{0}+a_{1} \cos \zeta$. It can be observed the parameter of thickness $a$ increases when the showers are early in age (large $\cos \zeta$ ). More evident is for the interval of $\sec \theta: 1.40$ to 1.60 which corresponds to the zenith angle of $44.4-51.3$ degrees. This effect called asymmetry in the air showers was also studied by other experiment [7].

\section{Summary}

By using the time profile of the TA-SD data the shower structure were studied using two observables. 1) The curvature of the shower front was studied in three different energy intervals. It is observed that one of the parameters of the shower front, A, depends on the zenith angle and the other parameter, B, has no dependence on the zenith angle but depends on energy. 2) The thickness 
of shower disk defined as the $10 \%-50 \%$ rise time was studied. Observing in a certain interval of $\mathrm{R}(500-1200 \mathrm{~m})$, it is found that the thickness of the shower disk shows a clear zenith angle dependence. It is also found that the parameter is not symmetric around the shower axis for early and late showers. All the analysis is based on the experimental data of the TA-SD array using 11 years of data (2008-05-11 to 2019-05-10).

\section{References}

[1] D. Ivanov, Energy spectrum measured by the Telescope Array surface detector, PhD Thesis, University of Utah (2012).

[2] T. Nonaka, et al. The surface detector array of the Telescope Array experiment Nuclear Instruments and Methods in Physics Research A, 689: 87-97 (2012).

[3] A.K Calabrese Malcarne, L. Perrone, A. Surdo, et al. Time structure of the extensive air shower front with the ARGO-YBJ experiment. Proceeding of the 31st ICRC, ŁÓDŹ, 689: 87-97 (2009).

[4] S. Yoshida, et al. The cosmic ray energy spectrum above $3 \times 10^{18} \mathrm{eV}$ measured by the Akenoi Giant Air Shower Array. Astroparticle Physics, 3:105-123 (1995).

[5] M. Teshima et al. The Properties of $10 * * 9-\mathrm{GeV}$ to $10 * * 10-\mathrm{GeV}$ Extensive Air Showers at Core Distances between 100-m and 300-m . J. Phys.,, G12:1097 (1986).

[6] Jhon Linsley and Livio Scarci Arrival times of air shower particles at large distances from the axis. Phys. Rev., 128: 2384-2392 (1962).

[7] A. Aab et al. Azimuthal asymmetry in the risetime of the surface detector signals of the Pierre Auger Observatory. Phys. Rev. D, 93: 072006 (2017).

[8] W. Hanlon et al. Deph of Ultra High Energy Cosmic Ray Induced Air Shower Maxima Measured by the Telescope Array Black Rock and Long Ridge FADC fluorescence Detectors and Surface Array in Hybrid Mode. The Astrophysical Journal, 858:76 (27pp) (2018). 